they have far fewer superposed impact craters than the cratered terrain?

Janes and Melosh suggest that the accretional collision could have melted some portion of Miranda's surface, which flooded over the sinker's site. Such melting would have to involve volatile ices (or perhaps the mobilization of methane clathrate ${ }^{8}$ ), as the low velocities noted above are not sufficient to melt water ice ${ }^{9}$. Perhaps a more plausible suggestion is that enhanced radiogenic heating by the silicate-rich sinker causes melting or mobilization of volatile ices at depth, which can then erupt to the surface. Melosh also suggests viscous relaxation could be involved, although there is no specific evidence for this (there are no partially relaxed craters, for example).

I suspect these lines of argument will turn out to be crucial to the acceptance or rejection of the sinker hypothesis. After all, resurfacing by volcanism is a natural component of the riser model. I would even go so far as to argue that the ridges in
Elsinore Corona (Fig. 1) are not tectonic at all, but are primary volcanic constructs, arcuate-to-linear extrusions of highly viscous ice lava complete with medial valleys. In addition, the discussion above has not touched on why sinkers or risers should operate in such a presumably cold and rigid body. Chaotic orbital dynamics and tidal heating are probably the key (see ref. 10), but that is another story.

1. Janes, D. M. \& Mekosh. H. J.J. geophys. Res. 93, 3127 3143 (1988).

2. Smith, B. A et al Science 215, 505-537 (1982).

3. Davis, D. R.. Farinella, P., Paolicchi, P. \& Zapallà, V Memorie Soc. astr. Ital. 57, 7-70)(1986).

Smith, B. A. et al. Science 233. 43-64 (1987).

Sohnson, T. V., Brown, R. H.\& Soderhlom, L. A. Scient Am. 256(4). 4R-60) (1987).

6. Thomas, P. C. lcuris 73, 427-441 (1988)

. Plescia. J. B. lcarus 73, 442-461 (1988)

8. Stevenson, D. J. \& Lunine, J. I. Nature 323, 46-48 (1986)

. Chapman $C, R$. \& McKinnon, W. B in Sutellites (ed Burns, J. A. \& Matthews, M. S.) 492-580 (University of Arizona Press. Tuscon. 1986)

10. Tittemore, W. C. \& Wisdom. J. Icarus (in the press).

William B. McKinnon is in the Department of Earth and Planetary Sciences and McDonnel Center for the Space Sciences, Washington University, Saint Louis, Missouri 63130, USA.

\title{
Malaria vaccines
}

\section{The shape of things to come}

\section{F. E. G. Cox}

RADICALLY new approaches to the development of a vaccine against malaria are likely to emerge as a result of two new discoveries. One suggests that aldolase, a key enzyme in glycolysis, produced during the maturation of the blood stages of the parasite, might be a possible target for immunological attack', and the other points to the possibility of delivering an oral vaccine against stages in the liver in transformed Salmonella ${ }^{2}$.

The life-cycle of the human malaria parasite Plasmodium falciparum is complex and involves an infective stage, the sporozoite; dividing stages in the liver and in the blood; and non-dividing sexual stages. Malaria parasites can reinfect humans repeatedly. With the increased resistance of the parasite to drugs like chloroquine and the failure of mosquitocontrol methods, the need for a vaccine against this devasting disease is critical.

Most of the research aimed towards developing malaria vaccines has concentrated on the surface antigens of the nondividing infective and sexual forms of the parasite and on the dividing forms in the blood. Several candidate antigens, all characterized by short repeated sequences of amino acids that provide an easy target for the immune response have been identified $^{3}$ and some have now been used in encouraging but not totally successful vaccination trials ${ }^{46}$. One problem with these antigens is that they could be diverting the immune system away from more vulnerable targets? such as enzymes.
Enzymes have frequently been considered as targets for chemotherapy but rarely as targets for immunological attack. But a group at the Hoffman la Roche Laboratories in Basel and the University Cantonal Hospital in Geneva, led by Ulrich Certa', has now identified malarial aldolase as one such target. Using standard gene-cloning techniques, the researchers have implicated a previously identified polypeptide of relative molecular mass 41,000, termed p41, associated with parasites maturing in the red blood cells. The $\mathrm{p} 41$ polypeptide has been shown to be partially protective against malaria in monkeys ${ }^{8}$, and is 60 per cent homologous to mammalian aldolase. The importance of this enzyme is that the blood stages of the malaria parasite lack a citric-acid cycle and so use vast amounts of glucose glucose uptake by infected cells is $10-100$ times that of uninfected cells". Aldolase catalyses the splitting of fructose-1.6-bisphosphate into glyceraldehyde 3-phosphate and dihydroacetone phosphate, thereby acting as a limiting step in glycolysis. The inhibition of aldolase activity would therefore totally inhibit the maturation of the parasite and subsequent invasion of fresh red blood cells.

There are several advantages of using aldolase as a target: it does not possess the repetitive sequences, characteristic of other malaria antigens, that might divert the immune response, and it does not exhibit allelic polymorphism thought to confer species or strain specificity on other candidate antigens. Thus there is a real possibility that aldolase could be a basis for a vaccine against many, if not all, types of malaria. On the other hand, the partial homology with human aldolase and the consequent possibility of inducing autoimmunity is a matter of some concern.

So far, all experimental vaccines against malaria have had to be injected, usually with an adjuvant. This is not only labour intensive and expensive but may even be dangerous in countries where the AIDS virus coexists with the multiple use of hypodermic needles. Jerald Sadoff and his colleagues at the Walter Reed Institute of Research in Washington, in collaboration with the Smith, Kline and French Laboratories, show ${ }^{2}$ that it is possible to develop an oral vaccine. They inserted the gene for the dominant antigen of the sporozoite. the infective stage injected by a mosquito, into attenuated Salmonella typhimurium which, when given orally to mice, in hibits the development of the liver stages. This occurs in the absence of antibody, confirming the growing opinion that immunity to these stages is cell-mediated. As genes for the human malaria parasite $P$. falciparum can be inserted into the human form of Salmonella. and as humans can respond to similarly transformed bacteria, there is no reason why a vaccine should not be developed. Again the advantages are that such a vaccine is unlikely to be species or strain specific.

What is the future for malaria vaccines? The conventional view of a cocktail of surface antigens from various stages of the parasite's life cycle is beginning to be replaced by the concept of another kind of cocktail. This would probably consist of antigens in transformed bacteria (or viruses) that would induce a cell-mediated response directed against the liver stages. together with enzyme-based antigens inducing antibody-mediated responses against target enzymes associated with the blood stages. In due course, antigens against the sexual forms could be added. It is also possible that antibodies against enzymes such as aldolase could be used to target drug delivery to predetermined components of the parasite's metabolism. The highly conserved nature of parasite enzymes renders them less likely to undergo selective changes resulting in drug resistance than more conventional chemotherapeutic targets.

\footnotetext{
1. Corta. U. en al Science 240. 10136-1038 (1988) Sadoff. J. at al Science' 240.336-335 (1988)

Kemp. D.J. Coppel. R.1 \& Anders. R.F. A. Res: Micro hinl 11. IS $-2(15)(1087)$

Billou W R ad Lamcet i, 1277-12\$1 (1987)

Herrington. D. A et al. Nature 328. 257-259 (1987)

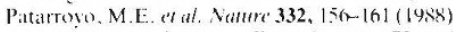

Anders. R.F. en al. Prog. Allergy 41. 148-172(1488). Perrin. L.H. et al. J. clin. Inve'st. 75. 1718-1721 (1985) WHO Tech Report Ser. No 743(1987).
}

F. E G Cox is Professor of Parasite Immunology in the Deparment of Biophysics. Cell and Molecular Biology. King's College London, 26-29 Drum Lane. London WC2B SRL. UK 